\title{
El registro de microvertebrados del sitio arqueológico Arroyo Malo 3 (Provincia de Mendoza, Argentina)
}

\author{
Record of microvertebrates from the archaeological site Arroyo Malo 3 \\ (Mendoza Province, Argentina)
}

GUSTAVO NEME1, GERMÁN MOREIRA², ADRIÁN ATENCIO \& LUCIANO DE SANTIS ${ }^{4}$

\author{
${ }^{1}$ Museo de Historia Natural de San Rafael, Parque Mariano Moreno s/n 5600 San Rafael, \\ Mendoza, Argentina \\ ${ }^{2}$ Comisión de Investigaciones Científicas de la Provincia de Buenos Aires (CIC), \\ 526 e/10 y 11, 1900 La Plata, Buenos Aires, Argentina \\ ${ }^{3}$ Facultad de Ciencias Exactas y Naturales, 5000 Córdoba, Argentina \\ ${ }^{4}$ Facultad de Ciencias Naturales y Museo, Paseo del Bosque s/n, 1900 La Plata, \\ Buenos Aires, Argentina; e-mail: desanti@movi.com.ar
}

\begin{abstract}
RESUMEN
En este trabajo se dan a conocer los resultados del análisis de microvertebrados registrados en el sitio arqueológico AMA-3, ubicado en el sur de la provincia de Mendoza. El lugar presenta una secuencia de ocupación que comienza hace 8.900 años AP. A lo largo de la misma se determinaron las distintas especies que habitaron la región durante el Holoceno y se las correlacionó con los cambios climáticos ocurridos durante este período. La presencia de Andalgalomys y Lestodelphys en diferentes momentos de la secuencia parece indicar la alternancia de diferentes momentos climáticos en esta región.
\end{abstract}

Palabras clave: microvertebrados, paleoambiente, Holoceno, Mendoza.

\begin{abstract}
Results are presented from the microvertebrates analysis of the archaeological site Arroyo Malo 3, located in Southern Mendoza. Different species were identified throughout the cultural sequence begining, 8,900 years BP. Correlations between the different microvertebrate species and Holocene climatic shifts are made. The oscilating presence of Andalgalomys and Lestodelphys at different intervals seen to indicate a regional Holocene climatic patern.
\end{abstract}

Key words: microvertebrates, paleoenvironment, Holocene, Mendoza

\section{INTRODUCCIÓN}

La creciente importancia en el estudio de los microvertebrados recuperados en el registro arqueológico ha sido puesta de manifiesto por numerosos investigadores (Pearson 1987, Andrews 1990, Stahl 1996). La metodología arqueológica con sus extracciones sistemáticas por nivel y su relación con la estratigrafía, permiten entender la distribución de las distintas especies, e interpretar así la historia de los elementos faunísticos en cada sitio. Pese a que diversos factores bioespeleológicos y posdepositacionales están incidiendo en la modificación del registro arqueológico en cada sitio, la presencia, ausencia y distribución de la fauna como bioindicadora, da claras pautas de los aspectos paleoambientales (Grayson 1984).
El presente trabajo tiene como objetivos, dar a conocer la lista sistemática de los microvertebrados hallados en el sitio Arroyo Malo 3 y a partir de estos discutir algunas inferencias paleoambientales. Para ello se realizaron observaciones tafonómicas que permitieron caracterizar el estado y origen de la muestra estudiada.

El sitio Arroyo Malo 3 es un alero basáltico que corresponde a la Formación Coyocho (Volkheimer 1975) y se encuentra ubicado en el sur de la provincia de Mendoza sobre el cauce cordillerano del Río Atuel, próximo a la desembocadura del Arroyo Malo ( $34^{\circ} 51^{\prime} 20^{\prime \prime} \mathrm{S}, 69^{\circ} 53^{\prime} 15^{\prime \prime} \mathrm{O}$ ) a unos $2.000 \mathrm{~m}$ de altitud (Fig. 1). Este sitio forma parte de una localidad arqueológica mayor dentro de la cual se encuentran ubicados otros cinco yacimientos. La secuencia de microvertebrados presentes en el sitio abarca un lapso temporal que se 
extiende desde el Holoceno temprano al Holoceno tardío. Existen muy pocos trabajos paleoclimáticos en el área, la mayoría de ellos se basan en estudios palinológicos y geológicos (D’Antoni 1983, Markgraf 1983) y estos muestran una continuidad en las características ambientales durante los últimos 4.000 años. El alto valle del Río Atuel también ha sido poco estudiado desde el punto de vista arqueológico y no existen antecedentes vinculados con el análisis de los microvertebrados; sin embargo sobre el cauce del Río Grande, al sur de la provincia y en el norte y sur del Neuquén, se han efectuado algunos aportes vinculados al tema (Pearson \& Pearson 1982, Pearson 1987, Massoia 1990, Neme et al. $1995^{1}$ ).

Los estudios se enmarcan en un plan de investigación para el cauce superior del Río Atuel, donde se proyecta avanzar en la caracterización de las adaptaciones de los grupos humanos a ambientes de altura. La alta antigüedad de la secuencia, la abundancia de restos de microvertebrados, la localización biogeográfica del sitio, las técnicas de recuperación empleadas y la buena conservación de los materiales resaltan la importancia de este sitio y su utilización en el estudio de las características climáticas del Holoceno. En este trabajo realizamos inferencias paleoambientales solamente para la zona en estudio; ya que para efectuar especulaciones a nivel regional se necesitaría de abundantes muestras sincrónicas (Pardiñas \& Lezcano 1995).

\section{MATERIALES Y MÉTODOS}

El sitio fue excavado en dos campañas sucesivas durante enero de 1995 y 1996, con un sondeo previo realizado en el verano de 1992. Durante los mencionados trabajos se abrió una cuadrícula de uno por dos metros, de donde se extrajeron 35 niveles artificiales de $5 \mathrm{~cm}$ hasta alcanzar la roca de base. Las excavaciones aportaron material cultural a lo largo de toda la secuencia, entre los que podemos mencionar elementos líticos, principalmente productos de talla, restos óseos y carbón. Si bien el análisis de los materiales culturales está en proceso, las ocupaciones humanas pertenecen a grupos de cazadores recolectores. La antigüedad de los restos eleva la explotación humana de la región al Holoceno temprano. A lo largo de toda la secuencia se nota un aprovechamiento diferencial de los recursos faunísticos y

${ }^{1}$ NEME G, V DURÁN \& A GIL (1995) Análisis arqueofaunístico del sitio Cueva de Luna (Malargüe, Mendoza). Actas del XII Congreso Nacional de Arqueología Chilena, Antofagasta. Chile: 363-370. líticos que evidencian cambios en las estrategias de aprovechamiento. En el primer caso, aunque se observan variaciones en los distintos momentos de ocupación del reparo, Lama guanicoe parece haber sido el principal recurso de subsistencia.

Desde el punto de vista fitogeográfico la zona corresponde al Dominio Andino, Provincia Patagónica (Cabrera 1971) y zoogeográficamente se encuentra ubicada en el Dominio Patagónico, Subregión Andino-Patagónica (Ringuelet 1961), muy próxima a la zona de ecotono con el Dominio Andino. Desde el punto de vista ecológico-geográfico el área en la que se encuentra el reparo corresponde a la denominada "Fauna de las montañas y la precordillera" (Roig 1972).

Las diferencias estacionales de precipitaciones y temperaturas, condicionadas por la altitud, tie-

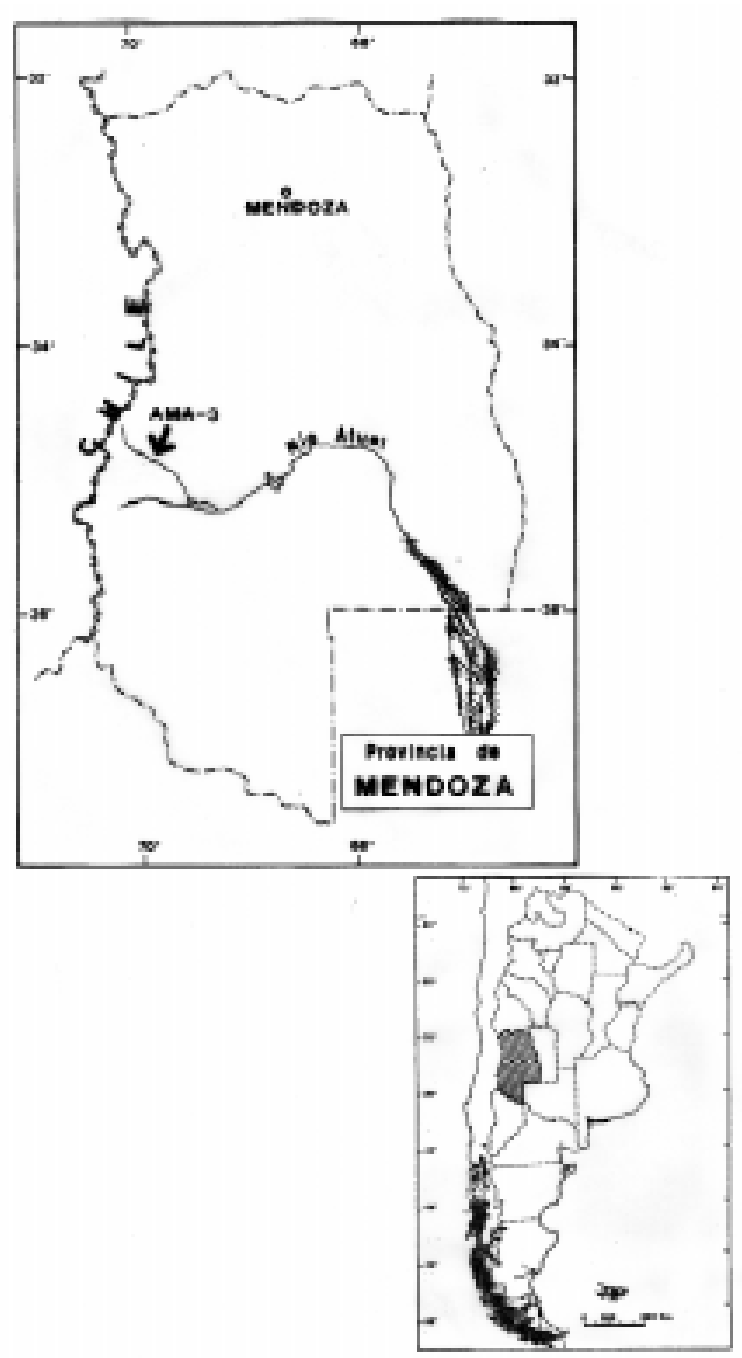

Fig. 1: Mapa de la provincia de Mendoza con la ubicación del sitio AMA-3.

Map of Mendoza Province showing the site Arroyo Malo 3. 


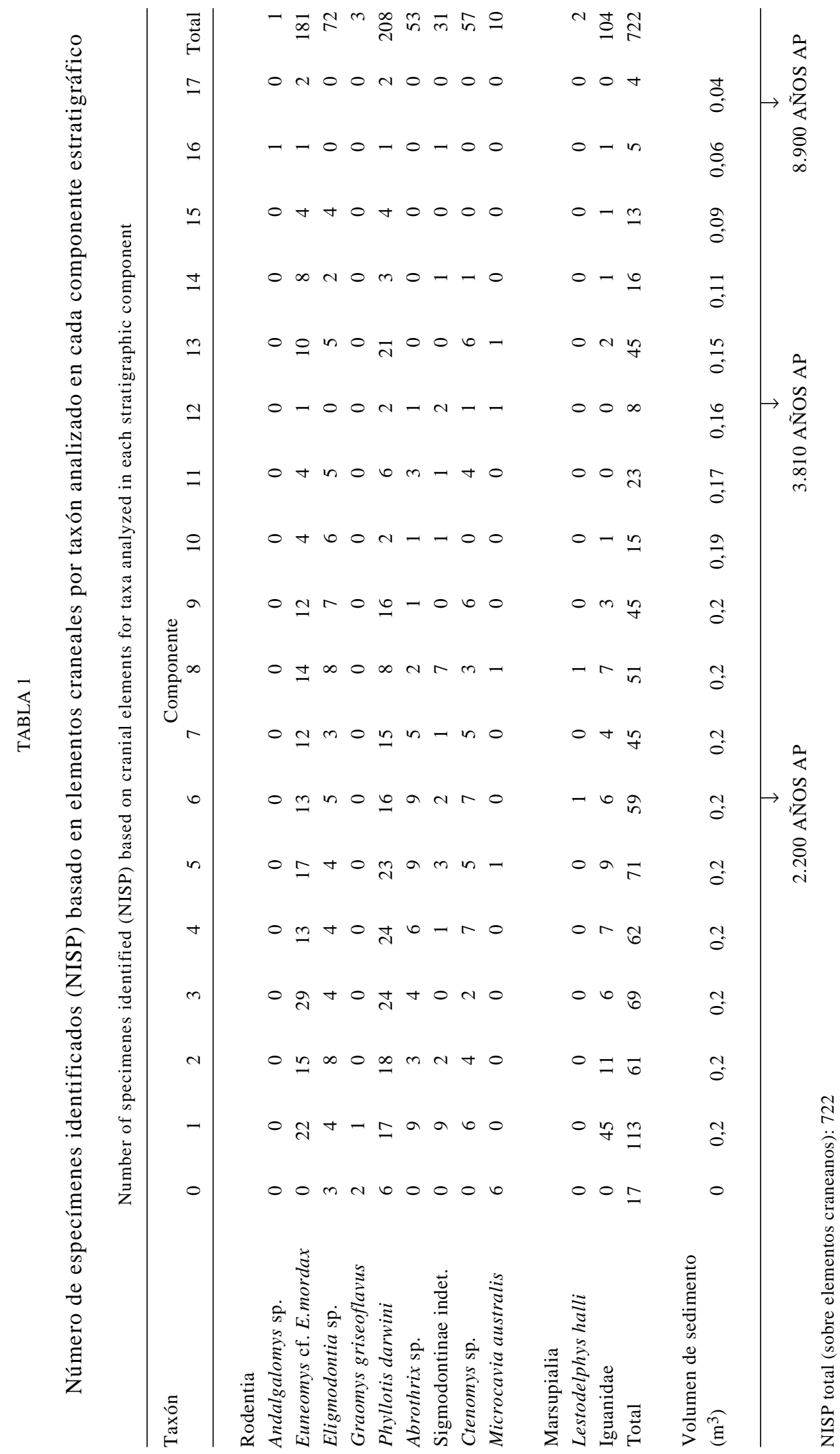


nen su correlato en las comunidades vegetales de la región. Así es que puede verse un cambio en las proporciones de las provincias fitogeográficas representadas a lo largo del valle del Río Atuel (Böcher et al. 1972). A éstas Roig (1972) las incluye dentro de las formaciones diversas de montaña que podían discriminarse de acuerdo a los niveles altitudinales, es así que desde unos 1.500 hasta $\operatorname{los} 2.300 \mathrm{~m}$ de altitud, existe una mayor proporción de elementos botánicos pertenecientes a la "Provincia Patagónica", que hasta los 1.800 se mezclan con componentes de la "Provincia del Monte" y desde los 2.300 hasta $3.800 \mathrm{~m}$ predominan especies de la "Provincia Altoandina" (Böcher et al. 1972).

Los 35 niveles artificiales fueron agrupados de a dos, a excepción del superficial que fue estudiado individualmente y los más profundos (33-34$35)$ se analizaron juntos, dando origen a 18 componentes. Los elementos extraídos fueron recuperados realizando mapeos tridimensionales y el sedimento fue cernido con una malla de $2 \mathrm{~mm}$ a fin de extraer la mayor cantidad de restos posibles. Además de los materiales culturales, se recolectaron durante la excavación todos los restos de microvertebrados y se determinaron fragmentos pertenecientes a otros vertebrados. A partir del nivel 20 (componente 10) la superficie de la excavación disminuye ya que la roca de base del alero comienza a avanzar paulatinamente hasta cubrir totalmente el piso de la excavación en el nivel 35 (componente 18). En la Tabla 1 se presentan conjuntamente las frecuencias taxonómicas con los cálculos de los distintos volúmenes de sedimento extraído por componente.

Las determinaciones se efectuaron sobre restos craneanos (maxilares y mandíbulas) según Bellocq \& Kravetz (1983); la lista sistemática se realizó siguiendo a Galliari et al. (1996). Para la determinación de las especies de Euneomys, nos basamos en el criterio de Pearson (1995), teniendo en cuenta solamente la longitud de la serie dentaria mandibular.

Se obtuvo el número mínimo de individuos (MNI) por componente y total. La frecuencia relativa de las distintas especies se estimó mediante el cálculo porcentual de cada una a partir de los elementos craneales presentes en cada componente; se estimó el porcentaje del MNI por especie. Estos valores se obtuvieron para los roedores sigmodontinos, los cuales son más representativos en la muestra (Tabla 2).

Para ubicar cronológicamente los restos recuperados se enviaron cuatro muestras de carbón de los niveles $12,24,31-32-33$ y 35, las cuales fueron datadas por radiocarbono en $8.900 \pm 60$ años AP (AA-26193), 7.670 \pm 100 años AP (LP-
783), $3.810 \pm 100$ años AP (LP-946) y $2.200 \pm 60$ años AP (LP-958). Con el objetivo de facilitar la visualización de posibles cambios en las frecuencias de microvertebrados a través del tiempo, se dividió en forma arbitraria la secuencia en tres conjuntos tomando como referencia los fechados obtenidos. El primero (A) abarca desde el inicio de la secuencia hasta los 2.200 años AP (componente 6), el segundo (B) desde 2.200 hasta 3.810 años AP (componente 12) y el tercero (C), desde 3.810 hasta 8.900 años AP (componente 18). Se obtuvo el NISP y la frecuencia por componentes (Tabla 3 y 4 ).

Los resultados de las determinaciones fueron discutidos en base a estudios previos similares realizados en sitios del sur de Mendoza (Neme et al. 1995), norte y sur del Neuquén (Pearson \& Pearson 1982, Massoia 1990) y centro de Chile (Simonetti 1989). Luego se realizaron comparaciones entre los conjuntos a lo largo de la secuencia para determinar cambios en las frecuencias de los distintos taxa, y su correlación con cambios paleoambientales.

Numerosos autores señalan los problemas relacionados con el tamaño de muestra que presenta el análisis de los distintos conjuntos arqueozoológicos (Grayson 1989, Kintingh 1989). Entre estos actualmente se acepta que existe una estrecha relación entre la diversidad de clases y el tamaño de la muestra. Esto implica que previo a discutir el significado de la diversidad taxonómica, debe considerarse su comportamiento con el tamaño de muestra. De esta forma puede evitarse considerar sesgos producidos por tamaños de muestra diferenciales. Por esto, antes de avanzar en la interpretación de los materiales subdivididos en los tres conjuntos, se correlacionó la diversidad con el tamaño de muestra para examinar si esta última podría ser la responsable de las diferencias en la diversidad observada entre estos conjuntos. Para tal fin se empleó el coeficiente no paramétrico de Spearman con una significancia de 0,05 . La correlación obtenida dio un valor alto $(0,9652)$ que es estadísticamente significativo en el nivel elegido, lo que indica que las diferencias en la diversidad entre los conjuntos está fuertemente influenciada por la cantidad de elementos recuperados en cada uno de ellos. Frente a la correlación detectada entre tamaño de muestra y diversidad y como un modo de considerar el problema, aplicamos un modelo de riqueza esperada basado en el tamaño de muestra (Kintingh 1989), a través de un programa de simulación desarrollado por Kintingh (1994) que es denominado DIVERS. Este programa permite analizar la diversidad controlando la influencia del tamaño de muestra. Antes de aplicarlo se calcularon los índices de riqueza y homogeneidad 


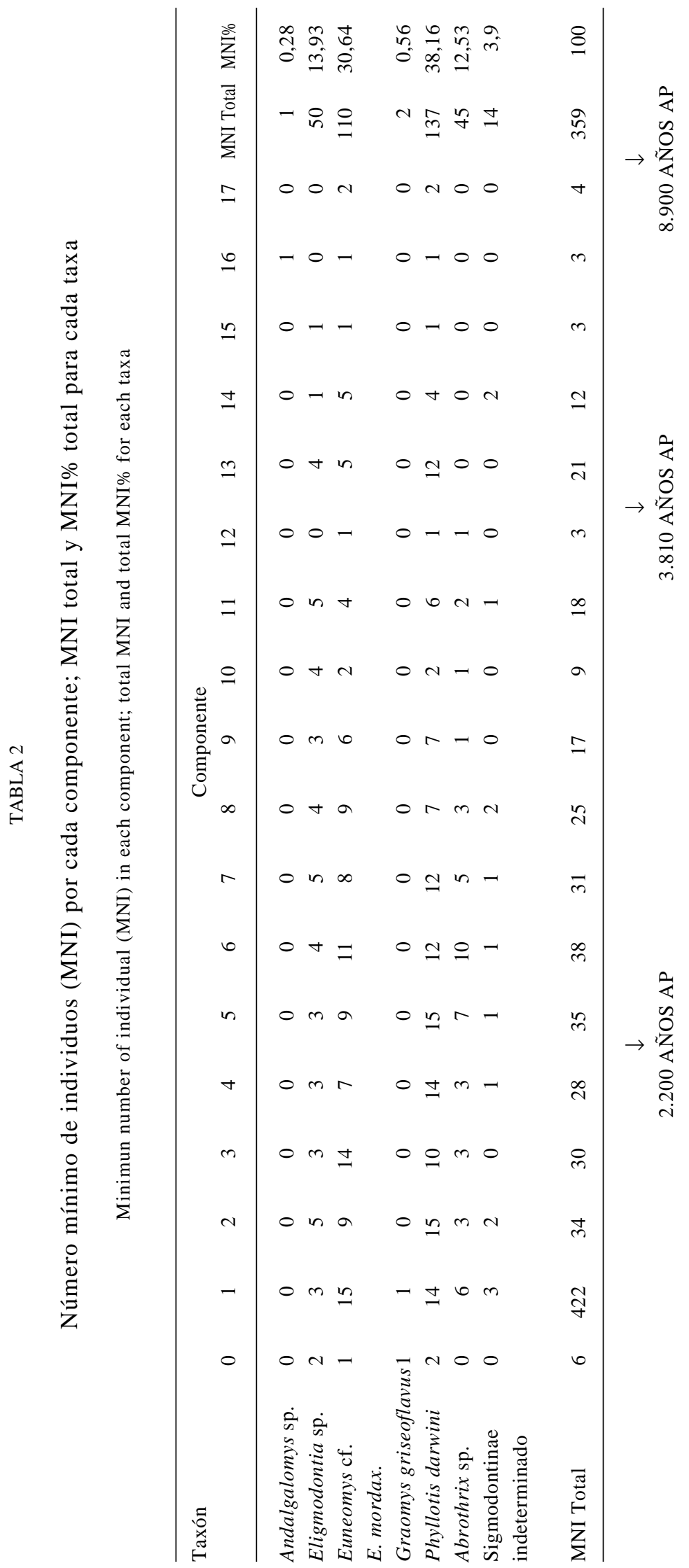


de Shannon y Weaver (ver Tabla 4). Las especificaciones y supuestos del programa están desarrollados por Kintingh (1989).

En el gráfico (Fig. 3) se puede ver que los tres conjuntos de Arroyo Malo 3 se encuentran dentro del rango de riqueza esperada con un intervalo de confianza. No se observa una riqueza significativamente más alta entre ninguno de los conjuntos. De esta forma se puede suponer que la riqueza entre éstos no ha sido afectada por el tamaño de muestra. Esto permitirá en adelante realizar inferencias sobre la diversidad observada en los distintos componentes del sitio Arroyo Malo 3.

\section{Agentes responsables de la acumulación de los} microvertebrados

La recuperación, determinación e interpretación de restos de microvertebrados en las excavaciones presenta problemas particulares, entre los que se pueden mencionar el pequeño tamaño del material, las dificultades en la recuperación completa de la muestra, problemas de determinación y la falta de estudios sobre el tema (Stahl 1996). Una de las principales dificultades en este tipo de análisis es interpretar cuál o cuales han sido los agentes responsables de la acumulación (Stahl 1996), y cómo afectan éstos la integridad de los depósitos. La principal causa de muerte entre los microvertebrados es la acción de depredadores que actúan de diversa forma de acuerdo al ambiente, hábitos de los mismos, de las presas y la demografía de las poblaciones de microvertebrados (Pearson 1985, Andrews 1990).

Para explicar el origen de la muestra debemos tener en cuenta una serie de rasgos propios de estos depósitos. Las acumulaciones de microvertebrados por acción de los carnívoros presentan características particulares que, en términos generales, no se ajustan a las de la muestra estudiada. Comúnmente en la fecas de éstos se puede observar una gran destrucción de las partes esqueletarias de las presas, fundamentalmente en lo que se refiere a cinturas y tibias por efecto de la masticación (Korth 1979). Por otro lado los huesos que no son destruidos en su totalidad presentan un alto grado de fractura y de corrosión en las epífisis y superficie, debido a la acción de los ácidos gástricos (Korth 1979, Stahl 1996).

En las rapaces diurnas (Accipitridae y Falconidae), si bien no producen una frecuencia tan alta de fracturas como los carnívoros, los ácidos estomacales corroen fuertemente las distintas partes del hueso, haciéndolo muchas veces no identificable (Korth 1979). Los porcentajes de representación de las partes esqueletarias son muy variables de acuerdo a la especie predadora, aunque son similares a los que presentan los mamíferos. Las rapaces nocturnas tales como búhos y lechuzas no dejan evidencias importantes de corrosión por ácidos estomacales, ni fracturas en los huesos largos. Todos los elementos esqueletales están presentes y los porcentajes de representación de las distintas partes no varían entre sí (Korth 1979). Si bien Andrews (1990) discute este grado de integridad a que hacemos referen-

TABLA 3

Número de especímenes (NISP) por conjumto estratigráfico recuperado en el sitio Arroyo Malo 3

Number of specimens (NISP) for stratigraphic asamblaje recovered in Arroyo Malo 3 site

\begin{tabular}{|c|c|c|c|c|}
\hline \multirow[t]{2}{*}{ Taxón } & \multicolumn{3}{|c|}{ Conjunto } & \multirow[b]{2}{*}{ Total } \\
\hline & A & B & $\mathrm{C}$ & \\
\hline Andalgalomys sp. & 0 & 0 & 1 & \\
\hline Euneomys cf. E. mordax & 109 & 47 & 25 & \\
\hline Eligmodontia sp. & 32 & 29 & 11 & \\
\hline Graomys griseoflavus & 3 & 0 & 0 & \\
\hline Phyllotis darwini & 128 & 49 & 31 & \\
\hline Abrothrix sp. & 40 & 13 & 0 & \\
\hline Sigmodontinae indeterminado & 17 & 12 & 2 & \\
\hline Ctenomys sp. & 31 & 19 & 7 & \\
\hline Microcavia australis & 7 & 2 & 1 & \\
\hline Lestodelphys halli & 1 & 1 & 0 & \\
\hline Iguanidae & 84 & 15 & 5 & \\
\hline Total & 452 & 187 & 83 & 722 \\
\hline
\end{tabular}


cia, el mismo autor reconoce que los niveles de fractura y corrosión producido por las rapaces nocturnas son los más bajos de todos los predadores. Saavedra \& Simonetti (1998) demostraron que dos poblaciones de Tyto alba producen diferentes conjuntos de huesos, y que las diferencias entre estos conjuntos pueden determinarse solamente a nivel ordinal.

La otra posibilidad que fue evaluada entre las causas de acumulación de los microvertebrados fue la actividad humana. El consumo de microvertebrados por el hombre, no solo ha sido registrado etnográficamente (Gusinde 1982), sino que también se ha afirmado desde una perspectiva arqueológica (Bond et al. 1981, Simonetti \& Cornejo 1991). Como señala Stahl (1996), si bien ha sido cuidadosamente tratado en la bibliografía, el consumo y/o utilización de roedores por parte de los grupos humanos es muy difícil de comprobar, debido a que las señales de actividad dejadas por el hombre en este tipo de restos puede ser producida por diversas causas. Entre los rasgos más notorios de actividad antrópica que podrían llegar a ser detectados, se pueden mencionar la alta frecuencia de huesos quemados, alto índice de fractura y alteración por ácidos gástricos, marcas de corte, alta concentración de huesos, preferentemente de las especies de mayor tamaño y algún tipo de estructura o contexto arqueológico distintivo de actividad humana relacionada al consumo y/o uso de microvertebrados (Bond et al. 1981, Simonetti \& Cornejo 1991, Stahl 1996).

El material estudiado se encuentra depositado en la Cátedra de Anatomía Comparada de la Facultad de Ciencias Naturales y Museo de la Universidad Nacional de La Plata.

\section{RESULTADOS}

\section{Tafonomía y estratigrafía}

Sobre las frecuencias taxonómicas a lo largo de la secuencia es necesario hacer algunas consideraciones tafonómicas que pueden explicar el origen de la muestra. Los sedimentos extraídos durante la excavación de los niveles son friables y poco compactos, lo que dificulta detectar la presencia de alteraciones posdepositacionales durante la excavación. Sin embargo se hallaron algunas posibles cuevas de roedores en los niveles artificiales $3,7,11,12$ y 14. Las dos primeras se ubicaban en el centro de las cuadrículas, las de los niveles 11 y 12 en los ángulos NE y SE y la última en la pared oeste. En todos los casos que se detectaron posibles alteraciones se tamizaron en forma separada los sedimentos que aparecían dentro de los
TABLA 4

Frecuencia relativa por conjuntos e índices de riqueza y homogeneidad. El coeficiente de correlación entre el tamaño de muestra y la riqueza de especies fue de 0,9652

Relative frecuency by assemblage and richness and homogeinity index. The coefficient of correlation between sample size and species richness was 0.9652

\begin{tabular}{lccc}
\hline Taxón & \multicolumn{3}{c}{ Conjunto } \\
& 1 & 2 & 3 \\
\hline Andalgalomys sp. & 0 & 0 & 1,2 \\
Euneomys cf. E. mordax & 24,11 & 25,13 & 30,12 \\
Eligmodontia sp. & 7,07 & 15,5 & 13,25 \\
Graomys griseoflavus & 0,66 & 0 & 0 \\
Phyllotis darwini & 28,31 & 26,2 & 37,34 \\
Abrothrix sp. & 8,84 & 6,95 & 0 \\
Sigmodontinae indeterminado & 3,76 & 6,41 & 2,4 \\
Ctenomys sp. & 6,85 & 10,16 & 8,43 \\
Microcavia australis & 1,54 & 1,06 & 1,2 \\
Lestodelphys halli & 0,22 & 0,53 & 0 \\
Iguanidae & 18,58 & 8,02 & 6,02 \\
& & & \\
Shanon-Weaver & 0,7548 & 0,7531 & 0,649 \\
Homogeneidad & 0,7549 & 0,7531 & 0,649 \\
\hline
\end{tabular}

sectores aparentemente alterados. Si bien estas han sido las únicas cuevas detectadas, debido a las características de los sitios excavados en reparos, no podemos descartar que las perturbaciones producidas por este tipo de vertebrados hayan sido mayores que las observadas (Durán 1991). Esto parece indicar que al menos una parte de los microvertebrados presentes puede haberse depositado por muerte natural mientras ocupaban sus cuevas y así haber introducido especímenes en la muestra estudiada.

En general los huesos de la secuencia presentan un buen estado de conservación y, si bien solo han sido identificados los elementos craneales, también está representado el resto del esqueleto. La muestra tampoco presenta rasgos de pisoteo, intemperización ni arrastre hídrico, tales como abrasión, selección de partes esqueletarias por tamaño y/o forma u orientación (Korth 1979, Andrews 1990).

La presencia del hombre y su actividad desarrollada dentro del alero podría ser tomada en cuenta como un factor que puede haber influido en la muestra del sitio, pero en este caso dicha actividad haría solamente disminuir la cantidad de microvertebrados dentro del registro y no alterar las frecuencias relativas entre los taxa. Esto pudo haber pasado en el componente 12 en el que la muestra disminuye considerablemente, siendo 
este el momento en que se producen las mayores tasas de depositación de restos culturales.

En resumen, la muestra de microvertebrados estudiada presenta un buen estado de conservación con pocos huesos fracturados, todas las partes esqueletales están presentes y no presentan rasgos de alteración por ácidos gástricos. En el caso de la secuencia de Arroyo Malo 3 no aparecen concentraciones de roedores que indiquen un aprovechamiento intensivo de los mismos. Los huesos tampoco presentan marcas y el único indicador que podría estar mostrando actividad humana es la presencia de un reducido número de huesos quemados (menos de 10), aunque desconocemos si ha sido en forma intencional. Todas estas características apoyarían la idea de que el conjunto fue depositado por acción de rapaces nocturnas y descartan la posibilidad de que hayan actuado otras especies de predadores presentes en la región. La presencia en los sedimentos de escasos restos de egagrópilas de Strigiformes compuestos principalmente por pelo, sustentan esta observación; éstos no fueron analizados debido a su estado fragmentario.

Características ecológicas de los taxa recuperados a lo largo de la secuencia

De acuerdo con Pearson \& Pearson (1982), la fauna corresponde a especies características de climas áridos, en su mayoría de la estepa patagónica. En la lista sistemática (Tabla 1), se observa un predominio de especies de ambientes xéricos (un $60 \%$ corresponden a especies típicas de ambientes xéricos, mientras que el resto son generalistas). Observando las frecuencias relativas de los taxa por componente se advierte que los mismos se mantienen más o menos constantes a lo largo de toda la secuencia, con ausencia y presencia en algunos componentes. En general, Euneomys cf. E. mordax y Phyllotis darwini cuantitativamente son los más abundantes; en el primer caso se trata de una especie que prefiere áreas rocosas y suelos arenosos (características propias del sitio estudiado), en el segundo caso se distribuye en ambientes con una mayor diversidad de habitats. Otro taxa bien representado es Eligmodontia, género que comparte la mayoría de las caracteristicas ambientales descriptas para Euneomys cf. E. mordax.

Según el bosquejo zoogeográfico de Ringuelet (1961), se observa que Andalgalomys se distribuye en Argentina por el Dominio Central y Lestodelphys en el Patagónico. Esta distribución disyunta es coincidente con el esquema fitogeográfico de Cabrera (1971), donde el pri- mero es endémico del Dominio Chaqueño y el segundo del Dominio Andino. El marmosino Lestodelphys halli, al igual que el género Andalgalomys se encuentra pobremente representado y no se ha hallado en los mismos segmentos temporales, lo que es coherente con las diferencias que presentan en su distribución actual. Es significativo resaltar que este último género constituye la cita más austral y el primer registro documentado para el Holoceno temprano (Fig. 2).

Los sigmodontinos G. griseoflavus y E. typus han sido hallados en egagrópilas de Athene cunicularia en la Reserva de Ñacuñán (Pescetti \& Monge 1994). Saba et al. (1995) sostienen que $G$. griseoflavus muestra mayor afinidad por zonas alteradas, mientras que E. typus tiene un patrón de distribución uniforme y Pearson et al. (1987) determinó que E. morgani disminuye notablemente con el sobrepastoreo. La aparición de $G$. griseoflavus en los componentes 0 y 1 (Tabla 1) estaría vinculada a la mayor afinidad de esta especie con ambientes alterados. Un caso similar podría ser el de Microcavia australis, que si bien se distribuye en ambientes rocosos, arenosos y secos, también habita áreas disturbadas por acción del hombre. El caso de estas últimas dos especies es coherente con las características que presenta la región en la actualidad, producto de la acción antrópica. Si bien los iguánidos en general son abundantes en ambientes desérticos, la imposibilidad de determinar a nivel específico los restos recuperados dificulta su utilización como indicadores ambientales.

\section{DISCUSIÓN}

\section{Los microvertebrados y el paleoclima regional}

Las especies de vertebrados recuperadas en sitios arqueológicos son usualmente utilizadas como indicadores de condiciones paleoambientales (Findley 1964); cambios temporales en la distribución y abundancia de taxa de vertebrados revelan la naturaleza del ambiente durante el tiempo de depositación (Graham 1976, Grayson 1977). Las bases para la realización de inferencias paleoambientales son los caracteres de las especies halladas en el registro arqueofaunístico (Simonetti 1989).

Si bien los estudios paleoclimáticos en el cauce del Río Atuel son muy escasos, es posible hacer algunas inferencias al respecto a través de la incorporación de estudios en áreas próximas. A partir de la observación de distintos indicadores, la totalidad de los trabajos muestran que han 
ocurrido sucesivos cambios desde principios del Holoceno (Lagiglia 1970, Heusser 1982², D’Antoni 1983, Markgraf 1983, Sting \& Garleff 1985, Espizua $1993^{3}$, Schäbitz 1994). Así podemos observar que durante el Holoceno medio ocurrieron las variaciones ambientales de mayor magnitud desde el retiro de los glaciares y que hacia el 3.000 años AP se instalaron las condiciones ambientales actuales (Lagiglia 1970, Heusser 1982, Markgraf 1983, Sting \& Garleff 1985).

Los análisis glaciológicos muestran que luego del decrecimiento glaciar ocurrido entre el 13.000 y 10.000 años AP se produce un avance para el Holoceno medio entre los 6.000 y los 4.000 años AP (Sting \& Garleff 1985). A través de estudios

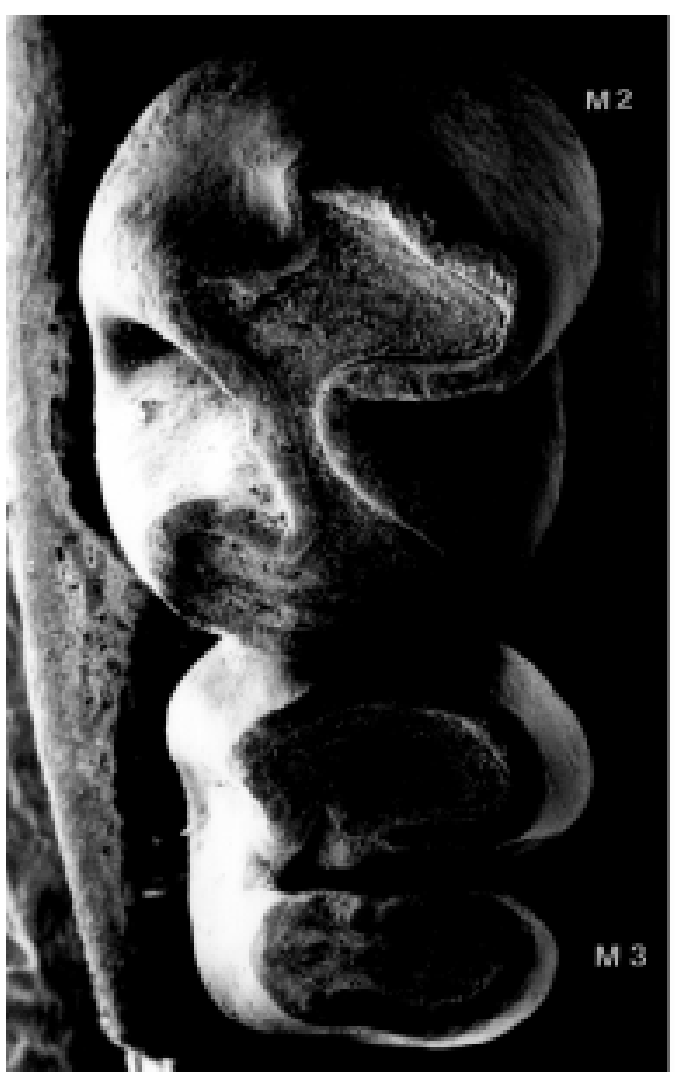

Fig. 2: Molares superiores derechos (M2 y M3) de Andalgalomys recuperado del componente 16 del sitio Arroyo Malo 3.

Right upper molars (M2 and M3) of Andalgalomys recovered in Arroyo Malo 3 site component 16.

${ }^{2}$ HEUSSER CJ (1982) Quaternary palynology of a core from Laguna de Tagua-Tagua in central Chile. Abstracts of the American Quaternary Association: 103.

${ }^{3}$ ESPIZUA L (1993) Glaciaciones cuaternarias. En: Ramos VA (ed) Geología y recursos naturales de Mendoza. Relatorio del XII Congreso Geológico Argentino, II Congreso Exploratorio de Hidrocarburos. palinológicos en la Cuenca del Salado Markgraf (1983) presenta datos coincidentes. Estos marcan un incremento de las precipitaciones invernales y un descenso de la temperatura para el mismo período.

Para Chile central, Heusser (1982), indica la presencia del altitermal con un clima frío y húmedo alrededor del 5.000 años AP que se corresponde con la neoglaciación evidenciada en Mendoza desde el 5.000 años AP (Espizua 1993). Entre los 8.500 y $\operatorname{los} 5.000$ años AP es posible notar a través de estudios polínicos un marcado incremento de la temperatura (D'Antoni 1983, Markgraf 1983). Por último todos los autores coinciden en que entre los 3.000 y 4.000 años AP se establecen las condiciones climáticas actuales (Lagiglia 1970, Markgraf 1983).

Las variaciones en el clima evidenciadas en avances y retrocesos de los glaciares, y cambios en la vegetación, parecen afectar menos de lo esperado a los registros de microvertebrados. Según lo muestran los distintos trabajos realizados por diversos autores, sería de esperar que estas alteraciones climáticas se correlacionen con desapariciones y apariciones de distintos taxa en el registro local; sin embargo sólo se observan pequeños cambios en las frecuencias de las distintas especies. Algo similar observan Simonetti \& Cornejo (1989) para Chile central. Pearson (1987) explica esto a través de las limitaciones que impone el registro paleobiológico, el cual no permite aislar períodos temporales cortos y por lo tanto no se pueden observar los cambios de algunas décadas o pocos siglos. Las variaciones observadas en las frecuencias podrían deberse a los cambios estacionales, pero esto es difícil de sostener debido a las características de recuperación del registro arqueológico.

Las especies presentes recuperadas en distintos sitios arqueológicos del centro oeste argentino, norpatagonia y Chile central muestran que para los últimos 8.000 años las condiciones ambientales habrían influido muy poco en la diversidad de los taxa, presentando distribuciones similares a las actuales (Pearson \& Pearson 1982, Pearson 1987, Simonetti 1989, Massoia 1990, Simonetti $\&$ Saavedra 1994, Neme et al. 1995). Esta constancia en la diversidad de los microvertebrados puede deberse a la falta de resolución temporal de las muestras analizadas, las cuales no permiten detectar fluctuaciones en los taxa de pocos siglos o varias décadas dentro de cada secuencia, en las cuales estarían reflejados los sucesivos avances y retrocesos glaciarios registrados a través de la geología y palinología (Pearson \& Pearson 1982).

Los trabajos realizados en áreas próximas como Chile central y norte del Neuquén con secuencias 


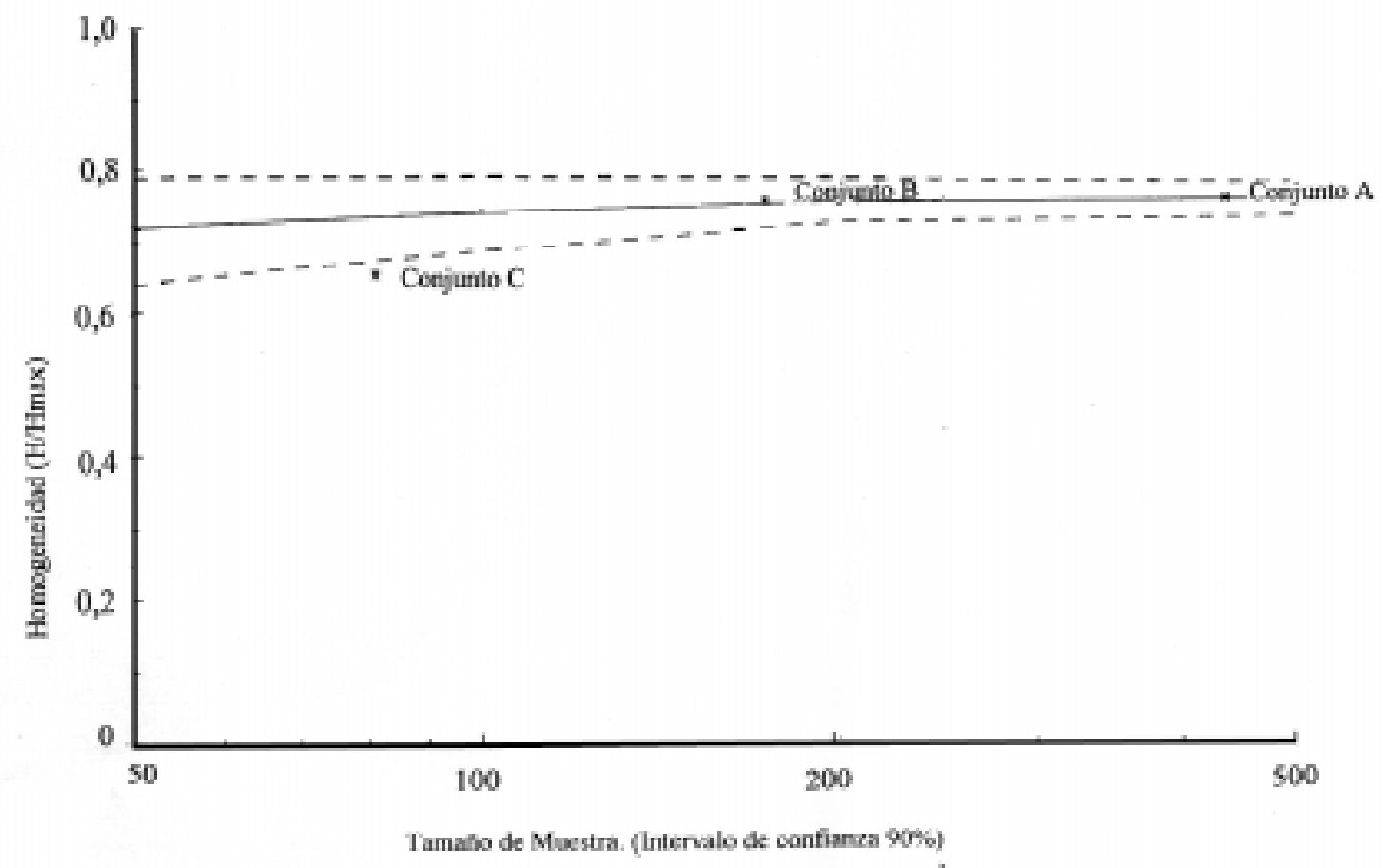

Fig. 3: Relación entre riqueza y tamaño de muestra.

Relationship between richness and sample size.

que en algunos casos abarcan lapsos de 10.000 años muestran que si bien el número de especies se ha mantenido constante a lo largo del tiempo, las diversidades han variado (Pearson 1987, Simonetti 1989, Massoia 1990, Simonetti \& Saavedra 1994, Neme et al. 1995).

Para discutir algunas de las hipótesis que aquí se presentan utilizamos los datos de abundancia relativa de cada especie por componente (Fig. 4), ya que aparentemente los cambios ambientales se reflejan mejor en este tipo de comparación y no a través de la presencia-ausencia de los taxa (Simonetti 1989). Esto puede deberse a que las variaciones en el clima no hayan sido lo suficientemente importantes como para afectar las distribuciones de cada especie a nivel de extinciones o invasiones de los taxa.

En general la diversidad y abundancia relativa de las especies no ha variado a lo largo del tiempo en los sitios arqueológicos estudiados, tanto para el sur de la provincia de Mendoza, como también en el norte y sur del Neuquén y Chile central. Algo similar ocurre en Arroyo Malo 3, sin embargo y si bien los índices de diversidad para los tres conjuntos no varían en forma significativa (ver Tabla 4), hay especies que se encuentran presentes en algunos de los componentes y en otros no.

Si bien como se mencionó anteriormente Lestodelphys halli y Andalgalomys corresponden a regiones biogeográficas diferentes, ambos géneros han sido hallados en el sitio Arroyo Malo 3. Sin embargo su ubicación estratigráfica es diacrónica, existiendo una diferencia temporal de alrededor de 4.000 años. En la actualidad el registro de microvertebrados es coherente con el área ecotonal donde se ubica el sitio.

En la secuencia analizada correspondiente a los componentes más antiguos (7.670 AP) se observa la presencia de Andalgalomys. Esto podría sustentarse en un aumento de la temperatura evidenciado en el último retroceso glaciar (Markgraf 1983), mientras que en los niveles superiores de la secuencia (3.800 años AP), se habrían establecido las condiciones climáticas actuales evidenciadas por la presencia de Lestodelphys. El brusco aumento en la frecuencia relativa de los iguánidos (Tabla 1 y Fig. 4), característicos de climas xéricos, a partir de los 4.000 años AP confirman esta idea de un aumento de la temperatura y un proceso de desertificación creciente para esta misma fecha. Mas allá de las variaciones mencionadas anteriormente, no se han podido registrar las alteraciones producidas durante el Holoceno medio (6.000 y 4.000 años AP), donde supuestamente se habría producido un período de enfriamiento de las condiciones locales producto del avance glaciar. Para los 4.000 años AP, se observa una mayor actividad humana dentro del 


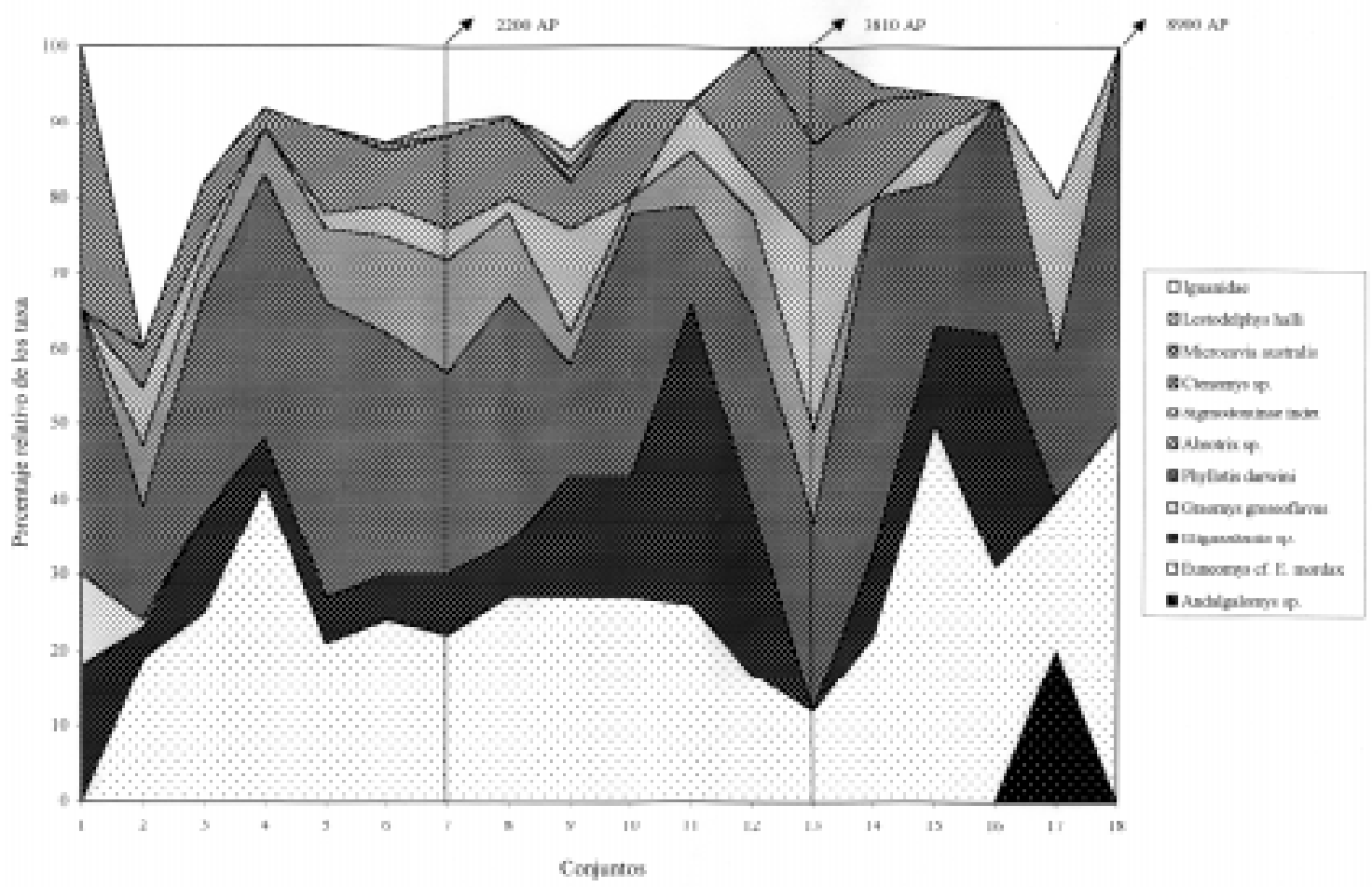

Fig. 4: Frecuencia relativa de los taxa por conjunto.

Relative frequency of taxa per aggregate unit.

alero, lo que podría ser interpretado como un indicador de mejores condiciones ambientales, las que favorecieron la explotación del área por parte del hombre.

\section{CONCLUCIONES}

La muestra analizada presenta buenas condiciones de conservación y todo indica que ha sido depositada por acción de Strigiformes. Si bien existe una fuerte correlación entre tamaño de muestra y diversidad, el tratamiento con modelos de riqueza esperada muestra que no ha sido afectada en forma importante.

El registro de los microvertebrados refleja que desde principios del Holoceno hasta fines del mismo, han ocurrido algunas variaciones en el número y cantidad de especies y también en la abundancia relativa de las mismas. Comparando estos cambios con los observados en el paleoclima regional y que han sido obtenidas a través de otros indicadores vemos que se comportan en forma muy similar (Fig. 4).

Así tenemos la presencia de Andalgalomys dentro del componente 16 (ca. 9.000 años AP) que para el comienzo de la secuencia se correlacionaría con un período más cálido. Este micromamífero es característico del Dominio Chaqueño. En los segmentos temporales posteriores al 4.000 años AP el registro de Lestodelphys evidenciaría la instalación de las condiciones climáticas actuales con un tipo de ambiente patagónico. El aumento en la frecuencia relativa de los Iguanidae a partir del 4.000 años AP sustenta, también, la instalación de las condiciones climáticas actuales.

Por último, la presencia de G. griseoflavus y Microcavia australis en los dos primeros componentes de la secuencia indicaría la sobrexplotación del ambiente por parte del hombre a través del sobrepastoreo.

La ampliación del registro de microvertebrados en el área para este tipo de análisis es posible gracias a la gran cantidad de reparos con características similares que se encuentran en la región y la posibilidad de ampliar las excavaciones en el reparo Arroyo Malo 3. Se espera que los trabajos futuros en el área puedan contribuir para contrastar las distintas ideas presentadas.

\section{AGRADECIMIENTOS}

Los autores del trabajo quieren agradecer especialmente a Arizona AMS Facility por la colaboración en la realización del fechado, a la Municipalidad de San Rafael por el apoyo brindado en 
los trabajos de campo, a Adolfo Gil por su asesoramiento en estadística y lectura crítica del manuscrito, a la Familia Vargas, quienes nos permitieron trabajar el área y colaboraron durante nuestra estadía en el Arroyo Malo, a Miguel Giardina por su colaboración durante los trabajos de excavación, a Humberto Lagiglia y Paula Novellino quienes leyeron versiones previas del manuscrito y a Brian Mceneaney por su colaboración con el inglés. Este trabajo fue parcialmente solventado con subsidios del CONICET (PICT-0353/96) y Fundación Antorchas.

\section{LITERATURA CITADA}

ANDREWS P (1990) Owls, caves and fossils. University of Chicago Press, Chicago, Illinois. 231 pp.

BELLOCQ MI \& FO KRAVETZ (1983) Identificaciones de especies, sexo y edad relativa a partir de restos óseos de roedores de la provincia de Buenos Aires, Argentina. Historia Natural (Argentina) 3: 101-112.

BOND M, S CAVIGLIA \& L BORRERO (1981) Paleoetnozoología del Alero de los Sauces (Neuquén, Argentina) con especial referencia a la problemática presentada por los roedores en sitios patagónicos. Instituto de Ciencias Antropológicas, Facultad de Filosofía y Letras, Universidad de Buenos Aires, Trabajos de Prehistoria (Argentina) Publicación 1: 93-109.

BÖCHER TW, P HJERTING \& K RAHN (1972) Botanical studies in the Atuel valley area, Mendoza Province, Argentina. Volume I, II \& III. Dansk Botansk Arkiv, Bind 22, No. 3. Kobenhavn, Dinamarca. 358 pp.

CABRERA AL (1971) Fitogeografía de la República Argentina. Boletín de la Sociedad Argentina de Botánica 14: 1-42.

D'ANTONI H (1983) Pollen analysis of Gruta del Indio. En: Rabassa J (ed) Quaternary of South América and Antartic Peninsula: 83-104. A.A. Balkema, Rotterdam, Holanda.

DURÁN V (1991) Estudios de perturbación por roedores del género Ctenomys en un sitio arqueológico experimental. Facultad de Filosofía y Letras, Universidad Nacional de Córdoba, Revista de Estudios Regionales (Argentina) 7: 7-31.

FINDLEY JS (1964) Paleoecologic reconstruction: vertebrate limitations. En: Hester JJ \& J Schoenwetter (eds) The reconstruction of past environments. Fort Burwing Research Center, Publication 3: 23-25.

GALLIARI C, UFJ PARDIÑAS \& FJ GOIN (1996) Lista comentada de los mamíferos argentinos. Mastozoología Neotropical (Argentina) 3: 39-61.

GRAHAM RW (1976) Late Winsconsin mammalian faunas and environmental gradients of the eastern United States. Paleobiology 2: 343-350.

GRAYSON DK (1977) Paleoclimatic implicationsof the Dirty Shame Rockshelter mammalian fauna. Tebiwa 9: 1-25.

GRAYSON DK (1984) Quantitative Zooarchaeology: topics in the analysis of archaeological faunas. Academic Press, Orlando, Florida. 202 pp.
GRAYSON DK (1989) Sample size and relative abundance in archaeological analysis: illustration from spiral fractures and seriation. En: Leonard J \& G Jones (eds) Quantifing diversity in archaeology: 5-24. Cambridge University Press, Cambridge, Inglaterra.

GUSINDE M (1982) Los indios de Tierra del Fuego. Tomo I, Volúmen 1. Centro Argentino de Etnología Americana, Consejo Nacional de Investigaciones Científicas y Técnicas, Buenos Aires, Argentina. 455 pp.

KINTINGH K (1989) Sample size, significance, and measures of diversity. En: Leonard R \& G Jones (eds) Quantifyng diversity in archaeology: 25-37. Cambridge University Press, Cambridge, Inglaterra.

KINTINGH K (1994) Tools for quantitative archaeology. Programs for quantitative analysis in archaeology. 145 pp. (unpublished manuscritpt)

KORTH WW (1979) Taphonomy of microvertebrates fossil assemblages. Annals of Carnegie Museum 48: 235285.

LAGIGLIA H (1970) Primer diagrama polínico de la estratigrafía arqueológica argentina. Reimpresión de Actas y Trabajos del Primer Congreso Nacional de Arqueología Argentina 11: 163-176.

MARKGRAF V (1983) Late and postglacial vegetational and paleoclimatic changes in subantartic and arid environments in Argentine. Palynology 7: 43-70.

MASSOIA E (1990) Zooarqueología 1 Mammalia. Anales de Arqueología y Etnología 2: 43-45.

PARDIÑAS UFJ \& M LEZCANO (1995) Cricétidos (Mammalia: Rodentia) del Pleistoceno tardío del nordeste de la provincia de Buenos Aires (Argentina): aspectos sistemáticos y paleoambientales. Ameghiniana 32: 249-265.

PEARSON OP (1985) Predation. En: Tamarin RH (ed) Biology of the New World Microtus. Special Publication of the American Society of Mammalogists 8: $535-566$.

PEARSON OP (1987) Mice and the postglacial history of the Traful valley of Argentina. Journal of Mammalogy 68: 469-478.

PEARSON OP (1995) Annotated keys for identifying small mammals living in or near Nahuel Huapi National Park or Lanín National Park, southern Argentina. Mastozoología Neotropical (Argentina) 2: 99-148.

PEARSON OP \& K PEARSON (1982) Ecology and biogeography of the southern rainforest of Argentina. En: Mares MA \& HH Genoways (eds) Mammalian biology in South America. Special Publication of Pymatuning Laboratory of Ecology 6: 129-142.

PEARSON OP, S MARTIN \& J BELLATI (1987) Demography and reproduction of the silky desert mouse (Eligmodontia) in Argentine. Fieldiana Zoology 39: 433-446.

PESCETTI E \& S MONGE (1994) Cadena alimentaria de la lechucita de las vizcacheras. Athene cunicularia. Pichiciego, Serie Monográfica 1: 1-8.

RINGUELET RA (1961) Rasgos fundamentales de la zoogeografía de Argentina. Physis (Argentina) 63: 151-170.

ROIG V (1972) Esbozo general del poblamiento animal en la provincia de Mendoza. Ministerio de Economía de la Argentina, Boletín de la Sociedad Argentina de Botánica 13 (Suplemento): 81-88. 
SAAVEDRA B \& JA SIMONETTI (1998) Small mammal taphonomy: intraspecific bone assamblage comparison between south and north american barn owl, Tyto alba, populations. Journal of Archaeological Science 25: $165-170$.

SCHÄBITZ F (1994) Holocene climatic variations in northern Patagonia, Argentina. Palaeogeography, Palaeoclimatology \& Palaeoecology 109: 287-294.

SIMONETTI JA (1989) Small mammals as paleoenvironmental indicators: validation for species of central Chile. Revista Chilena de Historia Natural 62: 109-114.

SIMONETTI JA \& LE CORNEJO (1991) Archaeological evidence of rodent consumption in central Chile. Latin American Anticuity 2: 92-96.

SIMONETTI JA \& B SAAVEDRA (1994) Reemplazando espacio por tiempo: arqueofauna del Estero del Manzano. Anales del Museo de Historia Natural de Valparaíso (Chile) 22: 113-119.
SABA SL, DA PÉREZ, E CEJUELA, V QUIROGA \& A TOYOS (1995) La piósfera ovina en el extremo austral del desierto del Monte. Naturalia Patagonica, Ciencias Biológicas 3: 153-174.

STAHL PW (1996) The recovery and interpretation of microvertebrate bone assemblages from archaeological contexts. Journal of Archaeological Method and Theory 3: 31-75.

STING \& GARLEF (1985) Glacier variations and climate of the late quaternary in the subtropical and midelatitude andes of Argentina. Aeitschrift für Gletscherkunde 21: 225-228.

VOLKHEIMER W (1975) Cerro Sosneado, Hoja $27 \mathrm{~b}$. Carta Geológico-Económica de la República Argentina, Ministerio de Economía, Secretaría de Estado de Recursos Naturales y Ambiente Humano, Subsecretaría de Minería, Servicio Geológico Nacional. 94 pp.

Editor Asociado: J.C. Torres

Recibido el 20 de diciembre de 1999; aceptado el 13 de noviembre de 2001 